\title{
CLEO-c Confronts High Precision Lattice QCD
}

\author{
Jim Napolitano*i \\ Rensselaer Polytechnic Institute \\ E-mail: napoljerpi.edu
}

\begin{abstract}
We discuss new results and upcoming measurements by the CLEO-c collaboration, on decays of heavy quark mesons and on the properties of heavy quarkonia. In particular, we emphasize those measurements which are just now becoming calculable in unquenched Quantum ChromoDynamics (QCD) on the lattice. Our ultimate goal is to provide a testing ground for recent approaches to "high precision" Lattice QCD.
\end{abstract}

XXIIIrd International Symposium on Lattice Field Theory

25-30 July 2005

Trinity College, Dublin, Ireland

\footnotetext{
*Speaker.

${ }^{\dagger}$ Representing the CLEO-c Collaboration
} 


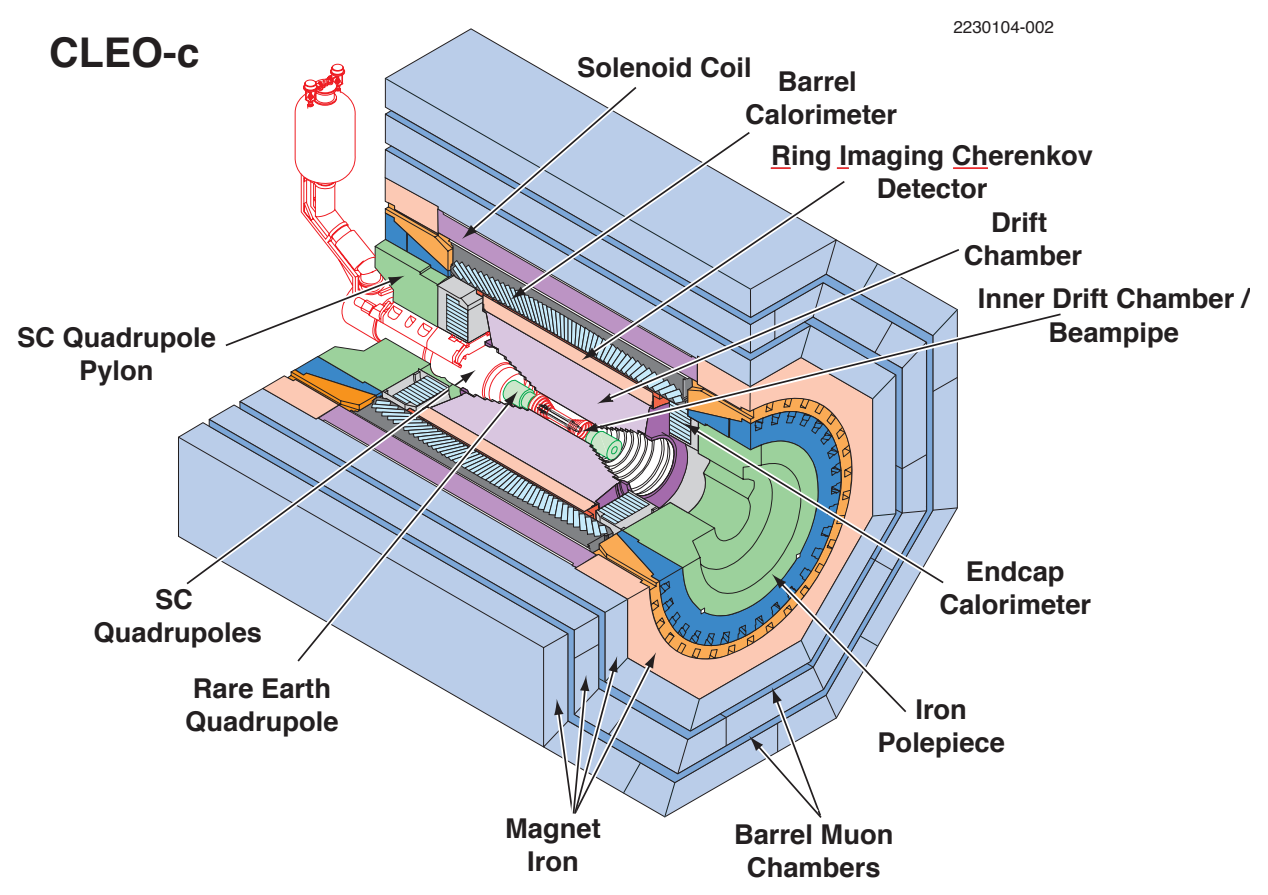

Figure 1: The CLEO-c detector at the Cornell Electron Storage Ring This is the latest incarnation of a detector with a more than two decade history of heavy quark physics in $e^{+} e^{-}$annihilation reactions.

\section{Introduction}

An experimentalist views Lattice Field Theory as a way to solve Quantum ChromoDynamics (QCD) so that its predictions can be compared to measurements. Historically [1] however, the precision with which one can make predictions has been far poorer than the corresponding experimental precision. One of the most important stumbling blocks has been the difficulty in doing calculations with small enough quark masses so that loops can be suitably included.

Recent advances [2, 3] using staggered fermions [4] have suggested that "high precision" $(\sim 1 \%)$ calculations may in fact be possible with presently available computing resources. Testing these predictions with measurements in heavy quark systems is a high priority for CLEO-c.

The CLEO experiment at Cornell University has been producing results for nearly 25 years [5], utilizing $e^{+} e^{-}$colliding beams at the CESR storage ring. The most recent version of the experiment to take data at high energy, CLEO III, included a new drift chamber [6] and a ring imaging Čerenkov detector for particle identification [7]. In addition to taking data at the $\Upsilon(4 S)$ for studies of $B$ mesons, the collaboration acquired several million events at each of the narrow $\Upsilon$ resonances, as well as other center of mass energies. Currently, the detector is in a configuration we call CLEO-c [8] to focus on physics in the charm threshold region. This is accompanied by a CESR upgrade to include an array of wiggler magnets which enhance luminosity for running at low energies. The CLEO-c detector is shown schematically in Fig. 1.

Running CLEO-c at lower energies, not far above the open charm threshold, allows us to make precision measurements of $D$ meson decay, even for rare processes. The key is that backgrounds can be greatly reduced when there are many kinematic constraints. For example, there is a large 


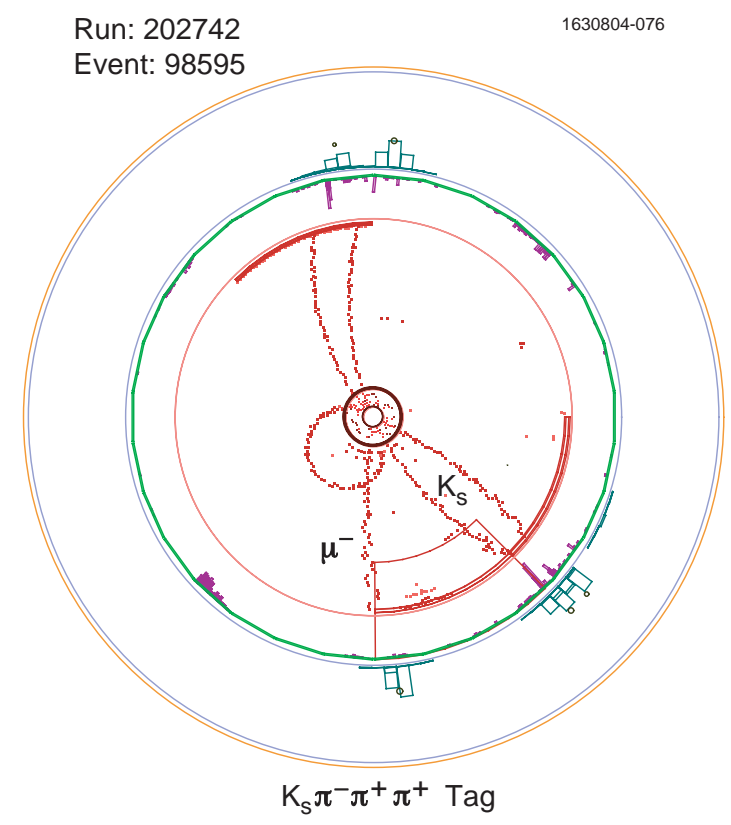

Figure 2: A CLEO-c event display, demonstrating the "tagging" procedure we use to make precision measurements with low background. This particular event shows the reaction $e^{+} e^{-} \rightarrow \psi(3770) \rightarrow$ $D^{+} D^{-}$, with $D^{+} \rightarrow K_{S} \pi^{+} \pi^{+} \pi^{-}$(and $K_{S} \rightarrow \pi^{+} \pi^{-}$) and $D^{-} \rightarrow \mu^{-} \bar{v}_{\mu}$. All tracks in the event are either $\pi^{+}$or $\pi^{-}$, except for the $\mu^{-}$which is labeled. Note that at this energy, the $D$ 's are each nearly at rest. The $D^{-}$is tagged with the $D^{+}$decay, where we observe all decay products of the latter. This completely kinematically constrains the $D^{-}$decay, and the unobserved neutrino is identified by virtue of its having the correct missing energy and momentum.

cross section for $e^{+} e^{-} \rightarrow D \bar{D}$ produced at the $\psi(3770)$. More importantly, however, there are no other channels open for bare charm at this energy. Therefore, the identification of a $D(\bar{D})$ implies the existence of a $\bar{D}(D)$ among the remainder of the event products. Furthermore, the momentum of one member of the pair determines the momentum of the other, while the energy of each must equal the energy of the beam. These are very powerful constraints, and the enable the precision measurements described in this talk.

These constraints are illustrated in the event display shown in Fig. 2. This event shows our observation of the rare process $D^{-} \rightarrow \mu^{-} \bar{v}_{\mu}$, from which ${ }^{1}$ we derive $f_{D^{+}}$. See Sec. 2 for details of the analysis. Although the details differ for other $D$ meson analyses, the principle is the same. Such is the basis for the contributions of CLEO-c to precision measurements for $D$ decay. ${ }^{2}$

We present the latest results from CLEO-c on purely leptonic decays of $D^{+}$mesons, the electronic widths of the narrow $\Upsilon$ resonances, and form factors in the semileptonic decays of $D^{+}$and $D^{0}$. Each of these supplies experimental quantities that are directly calculable in "high precision" Lattice QCD. We also review recent results from CLEO on the spectroscopy of heavy quarkonium. Finally, we discuss plans for the future, including planned measurements of $D_{s}$ meson decay and potential studies of glueballs.

\section{Latest results on $\mathbf{D}^{+} \rightarrow \mu^{+} v_{\mu}$}

Meson decay constants, $f_{X}$ for pseudoscalar meson $X$, essentially measure the overlap of the $q_{1} \bar{q}_{2}$ wave function in the meson, and are calculable to high precision in Lattice QCD [2]. They are determined from the purely leptonic decay mode $X^{+} \rightarrow \ell^{+} v_{\ell}$, which is helicity suppressed.

\footnotetext{
${ }^{1}$ Charge conjugation is assumed throughout this paper whenever relevant.

${ }^{2}$ At the conference, the speaker was asked to comment on CLEO's ability to contribute in this fashion to $B$ decay. Although the same ideas can in principle be applied to $B$ meson production at the $\Upsilon(4 S)$, the event rates are too low to be practical. In fact, CLEO no longer runs in this energy region.
} 


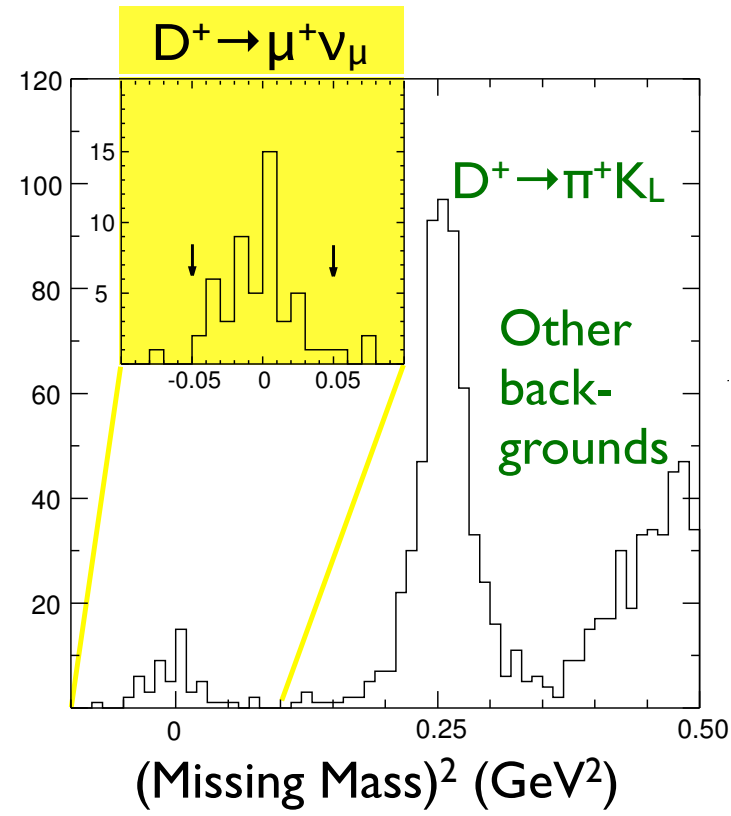

Figure 3: CLEO-c measurement of $D^{+} \rightarrow \mu^{+} v_{\mu}$. The figure histograms the (invariant) square of the the "missing mass", $M_{X}^{2}$, for the hypothesis $D^{+} \rightarrow$ $\mu^{+} X$ where $X$ is unobserved, in events where the $D^{-}$is fully reconstructed. This histogram shows our signal of fifty events, cleanly separated from the large number of $D^{+} \rightarrow \pi^{+} K_{L}^{0}$ and other backgrounds.

Therefore the rate is generally quite small, leading to small branching fractions and difficult measurements. The general expression for the decay rate is

$$
\Gamma\left(X^{+} \rightarrow \ell^{+} v_{\ell}\right)=\frac{G_{F}^{2}}{8 \pi} f_{X}^{2} m_{\ell}^{2} M_{X}\left(1-\frac{m_{\ell}^{2}}{M_{X}^{2}}\right)^{2}\left|V_{q_{1} q_{2}}\right|^{2}
$$

Using the techniques described above, CLEO-c has recently published a measurement of $f_{D^{+}}$ using the $\mu^{+} v_{\mu}$ decay of tagged $D^{+}$mesons [9]. That measurement used our first sample of data taken at the $\psi(3770)$ and was based on a signal of eight events.

CLEO-c has since accumulated $281 \mathrm{pb}^{-1}$ at the $\psi(3770)$ and a more precise result on $f_{D^{+}}$ is available [10]. Our signal is plotted in Fig. 3. We isolate the $D^{+} \rightarrow \mu^{+} v_{\mu}$ signal from backgrounds using the "missing mass" $M_{X}$, which is straightforward to determine given the kinematic constraints. The signal is the highlighted peak near $M_{X}^{2}=0$. The large peak near $M_{X}^{2}=0.25 \mathrm{GeV}^{2}$ is from $D^{+} \rightarrow \pi^{+} K_{L}$, where $\pi^{+}$are difficult to distinguish from $\mu^{+}$and the $K_{L}$ passes through the apparatus undetected. There are an estimated three background events in the signal region, denoted by vertical arrows in the inset.

From these data we find, for the branching ratio and decay constant,

$$
\begin{aligned}
\mathscr{B}\left(D^{+} \rightarrow \mu^{+} v_{\mu}\right) & =4.40 \pm 0.66_{-0.12}^{+0.09} \times 10^{-4} \\
f_{D^{+}} & =222.6 \pm 16.7_{-3.4}^{+2.8} \mathrm{MeV}
\end{aligned}
$$

where the first uncertainty is statistical and the second is systematic. More data taking is anticipated in the near future.

\section{Latest results on $\Gamma_{e e}$ for the narrow $\Upsilon$ resonances}

A very recent result from CLEO is now available, presenting the leptonic widths of the narrow bottomonium states. These preliminary values were just released at the time of the conference, and a public document describing the result in detail is not yet available. 

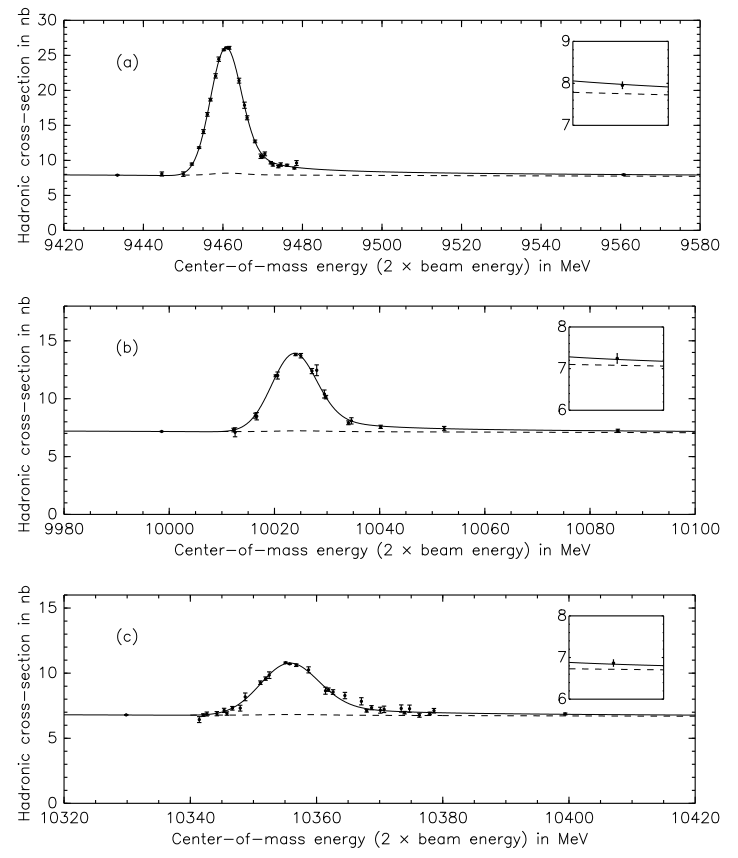

Figure 4: CLEO-III data for the total cross section for $e^{+} e^{-}$annihilation in the neighborhood of the (a) $\Upsilon(1 S$ ), (b) $\Upsilon(2 S)$, and (c) $\Upsilon(3 S)$ resonances. The solid line through the points is the result of a fit that includes a Breit-Wigner resonance shape, folded with the energy resolution of the beam and a radiative tail, along with various backgrounds. The dashed line shows the sum of all backgrounds alone, and the inset shows a close-up of the radiative tail region. These data are used to extract the rate for $e^{+} e^{-}$decay of these narrow $b \bar{b}$ states.

In the same way that a purely leptonic heavy meson $X$ decay such as

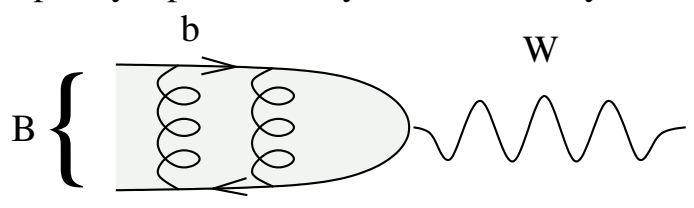

$\overline{\mathrm{u}}, \overline{\mathrm{d}}$

is sensitive to an observable, $f_{X}$, calculable in Lattice $\mathrm{QCD}$, so is the annihilation through a virtual photon for heavy quarkonium, for example

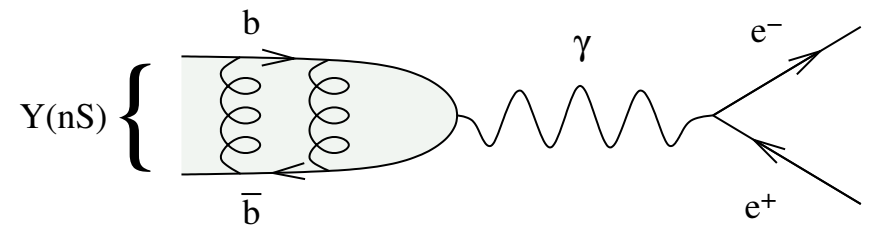

In this case, one measures the width of the quarkonium resonance to decay to an $e^{+} e^{-}$pair.

The CLEO collaboration, in the CLEO-III configuration, took a large sample of data in the region of, and at the peaks of, the three narrow $\Upsilon$ resonances, before changing over to run at the lower energies just above charm threshold, as CLEO-c. By making careful measurements of the total cross section for $e^{+} e^{-}$annihilation in the neighborhood of these resonances, it is possible to extract the width $\Gamma_{e e}$ for decay to $e^{+} e^{-}$pairs with high precision.

Our total cross section data is shown in Fig. 4. From these data, and from a careful determination of detection efficiencies, we find the following preliminary values for the electronic widths:

$$
\begin{aligned}
& \Gamma_{e e}(1 S)=1.336 \pm 0.009 \pm 0.019 \mathrm{keV} \\
& \Gamma_{e e}(2 S)=0.616 \pm 0.010 \pm 0.009 \mathrm{keV} \\
& \Gamma_{e e}(3 S)=0.425 \pm 0.009 \pm 0.006 \mathrm{keV}
\end{aligned}
$$


Once again, the first uncertainty is statistical and the second is systematic. The systematic uncertainties can be reduced if one considers instead ratios of these widths. We find

$$
\begin{aligned}
& \Gamma_{e e}(2 S) / \Gamma_{e e}(1 S)=0.461 \pm 0.008 \pm 0.003 \\
& \Gamma_{e e}(3 S) / \Gamma_{e e}(1 S)=0.318 \pm 0.007 \pm 0.002 \\
& \Gamma_{e e}(3 S) / \Gamma_{e e}(2 S)=0.690 \pm 0.019 \pm 0.006
\end{aligned}
$$

\section{Latest results on $\mathrm{D}^{+}$and $\mathrm{D}^{0}$ semileptonic decay}

The rate for semileptonic $D^{0}$ decay is given by

$$
\frac{d \Gamma}{d q^{2}}\left(D^{0} \rightarrow h^{-} e^{+} v_{e}\right)=\frac{G_{F}^{2}}{24 \pi^{3}} p_{h}^{3}\left|V_{c q}\right|^{2}\left|F\left(q^{2}\right)\right|^{2}
$$

where, for this discussion, we take $h=K(\pi), q=s(d), p_{h}$ is the momentum of the recoiling hadron, $V_{c q}$ is the relevant CKM matrix element, $q^{2}$ is the invariant mass of the $e^{+} v_{e}$ system, and $F\left(q^{2}\right)$ is the hadronic form factor at the $c \rightarrow W q$ vertex. The form factor can be predicted from a number of different theoretical approaches $[11,12,13]$ including Lattice QCD $[14,15]$. In addition to its own intrinsic interest, the analogous form factor is needed for extracting $b$-quark matrix elements such as $V_{u b}$ in $B$ semileptonic decays, so it is important to demonstrate the reliability of any one calculation.

Of all the $D^{0}$ semileptonic decays, $D^{0} \rightarrow \pi^{-} e^{+} v_{e}$ provides the greatest range of $q^{2}$. However, this is a challenging measurement because $\left|V_{c d}\right|^{2} \ll\left|V_{c s}\right|^{2}$ and there is a large potential background from $D^{0} \rightarrow K^{-} e^{+} v_{e}$. Experimenters must rely on good particle identification and/or kinematic constraints to reduce this background.

Here we once again demonstrate the power of CLEO-c, comparing it to measurements made with CLEO-III and charm production at high energy. Using $\sqrt{s} \sim 10 \mathrm{GeV}$ data, CLEO-III [16] identified $D^{0}$ mesons through the continuum production of $D^{\star^{+}} \rightarrow \pi_{\text {slow }}^{+} D^{0}$. The neutrino is reconstructed by identifying all other charged and neutral particles in the event and forming the effective $D^{\star^{+}}-D^{0}$ mass difference $\Delta M$, which is plotted on the left in Fig. 5. Backgrounds are clearly severe, especially the "peaking" background from $D^{0} \rightarrow K^{-} e^{+} v_{e}$ where the $K^{-}$is misidentified as a $\pi^{-}$. On the other hand, $D^{0} \rightarrow \pi^{-} e^{+} v_{e}$ is cleanly separated from all backgrounds using the kinematic constraints available in CLEO-c, as shown on the right in Fig. 5. In this case, the signal is seen as a peak in the difference between the missing energy and the missing momentum, determined using the semileptonic decays of $D$ 's that are "tagged" as described above.

Based on our first $56 \mathrm{pb}^{-1}$ sample from running at the $\psi(3770)$, CLEO-c has submitted for publication new values for semileptonic decays of $D^{0}[17]$ and $D^{+}[18]$ mesons. We find the following branching ratios (in per cent):

$$
\begin{array}{c|c|c|} 
& \multicolumn{1}{c}{D \rightarrow \pi e^{+} v_{e}} & \multicolumn{1}{c}{D \rightarrow K e^{+} v_{e}} \\
\cline { 3 - 4 } D^{0} & 0.262 \pm 0.025 \pm 0.008 & 3.44 \pm 0.10 \pm 0.10 \\
\cline { 2 - 3 } D^{+} & 0.44 \pm 0.06 \pm 0.03 & 8.71 \pm 0.38 \pm 0.37 \\
\cline { 2 - 3 } & &
\end{array}
$$



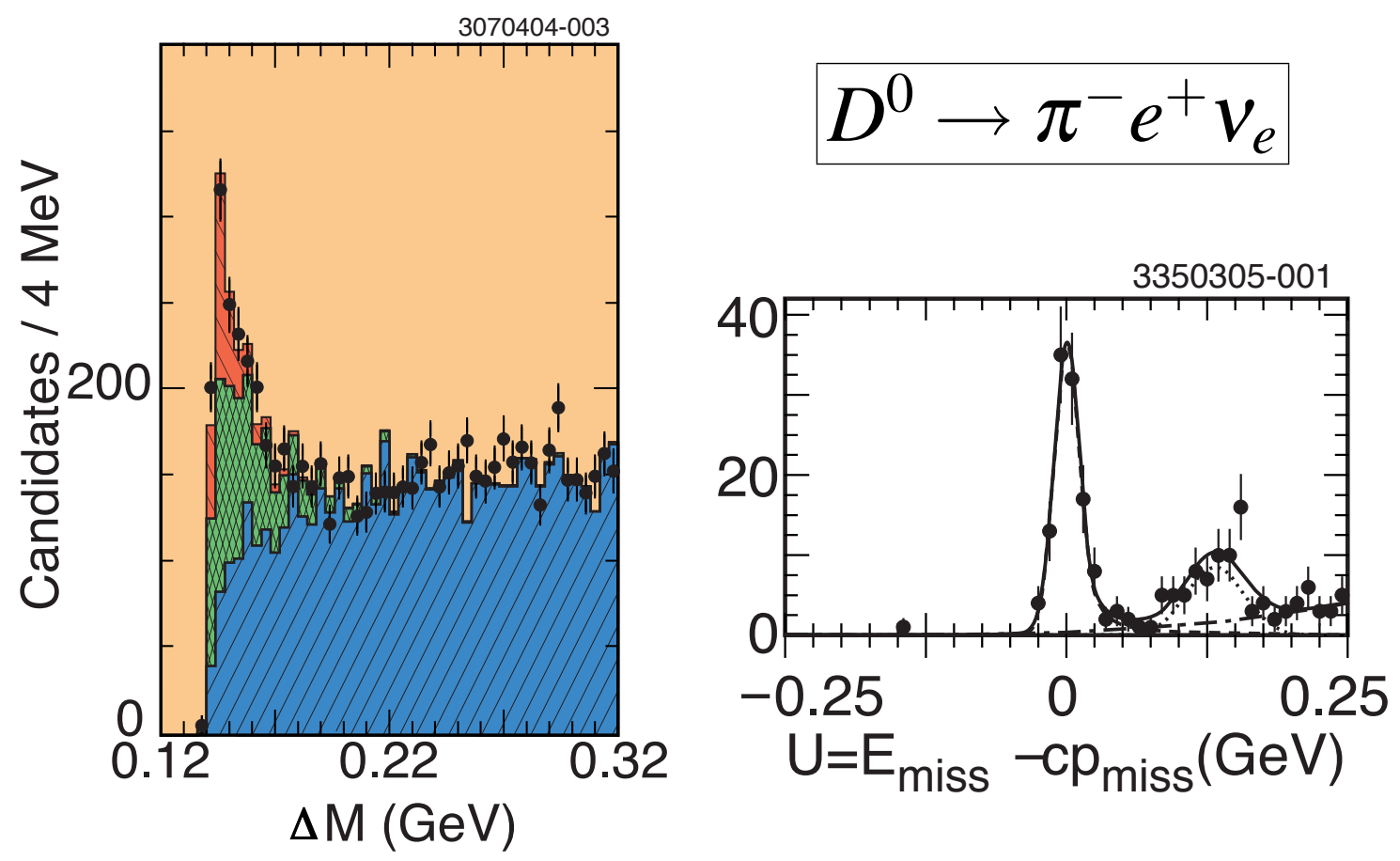

Figure 5: Comparison of signal and background for measurements of $D^{0} \rightarrow \pi^{-} e^{+} v_{e}$ in CLEO-III [16] (left), for $D^{0}$ 's produced on or below the $\Upsilon(4 S)$ resonance, and in CLEO-c [17] (right), for $D^{0}$ 's produced in correlated pairs at the $\psi(3770)$. The three hatched areas on the left indicate signal (upper, red), backgrounds from $D^{0} \rightarrow K^{-} e^{+} v_{e}$ (middle, green), and other backgrounds (lower, blue). For CLEO-c, on the right, the signal is contained in the isolated peak near $U=0$, and backgrounds are clearly very small.

Ratios can be derived from these measurements, decreasing the systematic uncertainties in some cases:

$$
\begin{aligned}
\mathscr{B}\left(D^{0} \rightarrow \pi^{-} e^{+} v_{e}\right) / \mathscr{B}\left(D^{0} \rightarrow K^{-} e^{+} v_{e}\right) & =0.076 \pm 0.008 \pm 0.002 \\
\Gamma\left(D^{0} \rightarrow K^{-} e^{+} v_{e}\right) / \Gamma\left(D^{+} \rightarrow \bar{K}^{0} e^{+} v_{e}\right) & =1.00 \pm 0.05 \pm 0.04 \\
\Gamma\left(D^{0} \rightarrow \pi^{-} e^{+} v_{e}\right) / 2 \times \Gamma\left(D^{+} \rightarrow \pi^{0} e^{+} v_{e}\right) & =0.75_{-0.11}^{+0.14} \pm 0.04
\end{aligned}
$$

Of course, these values are not directly testable with Lattice QCD because they are combined with the relevant CKM matrix elements. However, they demonstrate our precision in $D$ semileptonic decays, including integrating over the form factors. Separate results on the form factors themselves, based on our larger current data sample, are forthcoming.

\section{Summary of recent results on heavy quarkonia}

CLEO is also contributing to the spectroscopy of heavy quarkonia, including information on their internal radiative transitions. Results are highlighted here which have at least some bearing on modern calculations in Lattice QCD. These results are based on a sample of $3.1 \mathrm{M} \psi(2 S)$ and of $5.8 \mathrm{M} \Upsilon(3 S)$ taken with the CLEO-III and CLEO-c detectors. 

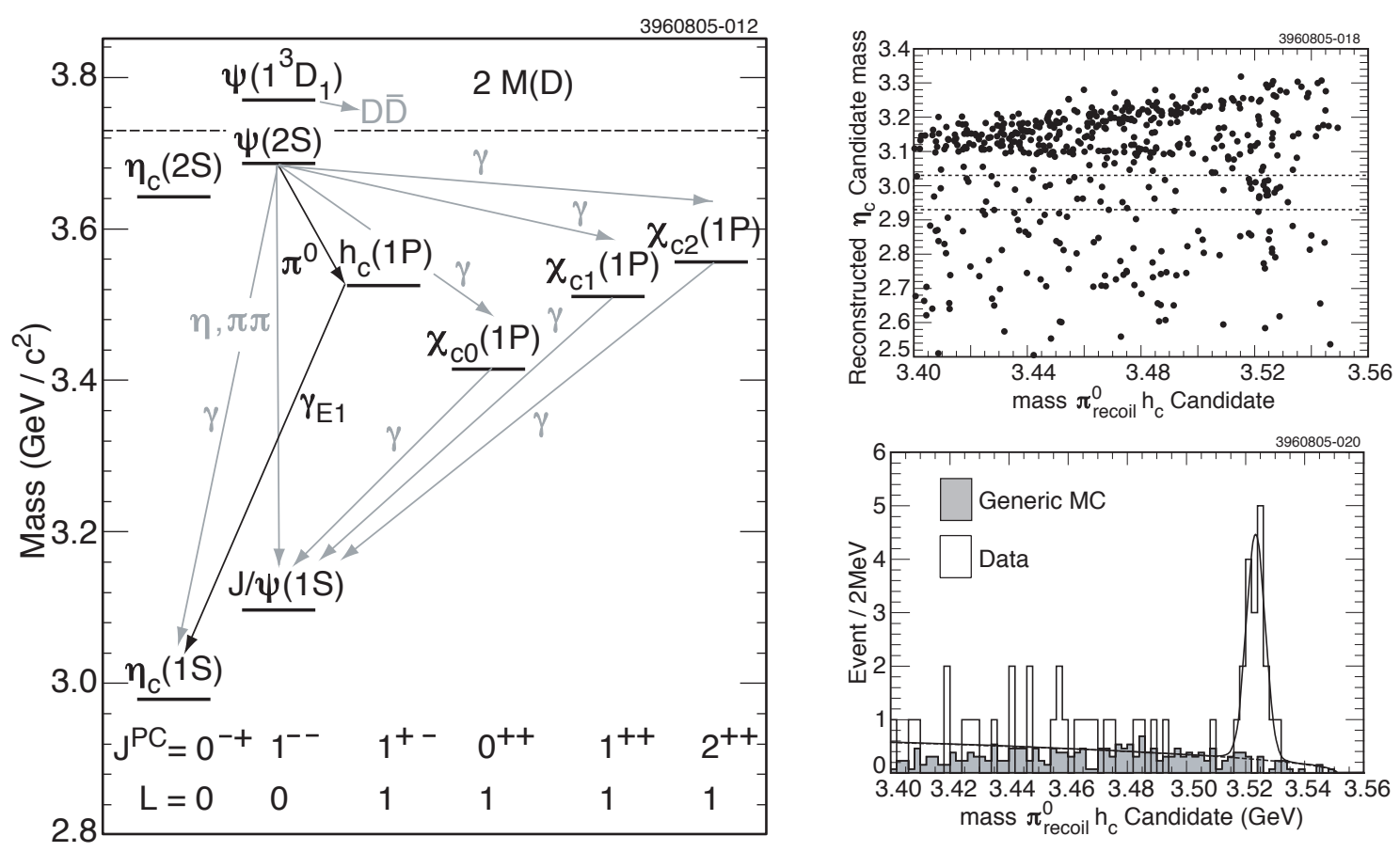

Figure 6: Charmonium and the $h_{c}$. The level diagram on the left shows our current understanding of the states below and near $D \bar{D}$ threshold. The isospin-violating decay $\psi(2 S) \rightarrow \pi^{0} h_{c}$ is highlighted. Various radiative transitions are also shown. On the right we plot details of our search for exclusive $h_{c}$ production in $\psi(2 S)$ decay. The $\eta_{c}$ is reconstructed using one of several decay modes, and the $\pi^{0}$ is also detected, and the mass against which it recoils is used to make the scatterplot, and is also histogrammed after cutting on the $\eta_{c}$ mass. A Monte Carlo simulation, based on generic $\psi(2 S)$ decays, demonstrates that the peak at $3.52 \mathrm{GeV}$ is not an artifact. CLEO has also observed the $h_{c}$ inclusively, that is, requiring the photon in the $h_{c} \rightarrow \gamma \eta_{c}$ transition but not observing the $\eta_{c}$.

\subsection{Observation of the $1^{1} P_{1}$ charmonium state $h_{c}$}

Figure 6 shows the level diagram for the $c \bar{c}$ system, mainly below $D \bar{D}$ threshold. Until recently, the ${ }^{1} P_{1}$ state, called the $h_{c}$, had been unidentified. Unlike the $\chi_{c_{J}}$ states, selection rules forbid radiative transitions to it from the $\psi(2 S)$. It can be reached via $\pi^{0}$ emission, but this decay violates isospin and is therefore quite suppressed.

CLEO-c has observed $[19,20]$ the $h_{c}$ using the $\psi(2 S)$ sample, in both exclusive (where the $\eta_{c}$ from $h_{c} \rightarrow \gamma \eta_{c}$ is reconstructed) and inclusive $\left(\eta_{c} \rightarrow X\right)$ final states. We determine the mass

$$
M\left(h_{c}\right)=3524.4 \pm 0.6 \pm 0.4 \mathrm{MeV}
$$

and therefore the hyperfine splitting with the weighted ${ }^{3} P_{1}$ states

$$
\Delta M_{\mathrm{HF}}(1 P)=1.0 \pm 0.6 \pm 0.4 \mathrm{MeV}
$$

\subsection{First observation of an $\Upsilon(1 D)$ state}

Figure 7 shows the level diagram for the $b \bar{b}$ system. CLEO has observed [21] the $\Upsilon(1 D)$ state for the first time, through the four photon cascade indicated in the figure. Different methods are 

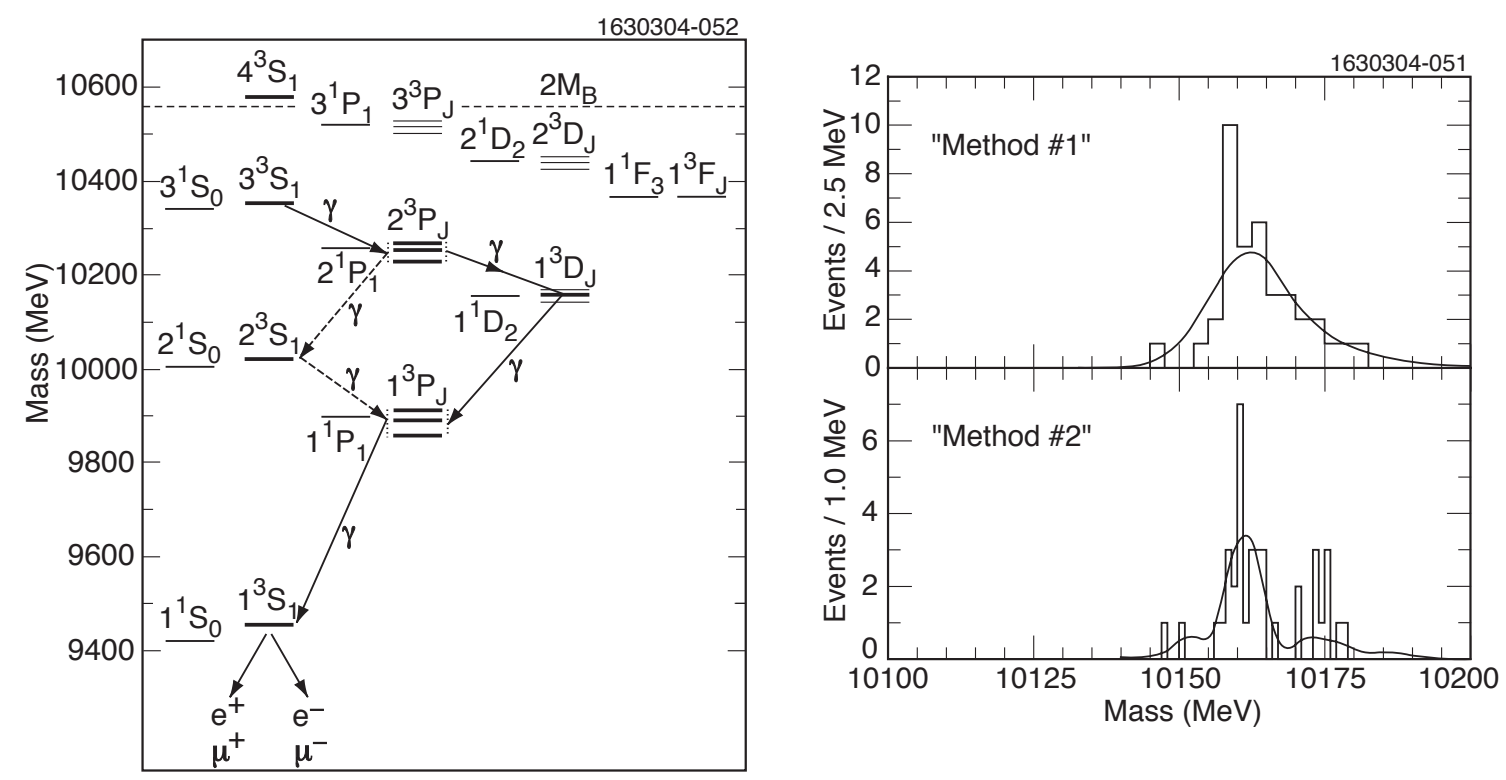

Figure 7: Bottomonium and the $\Upsilon(1 D)$. The level diagram is shown on the left, including the four-photon cascade in which the $\Upsilon(1 D)$ triplet is reached from the $\Upsilon(3 S)$, and subsequently decays reaching the $\Upsilon(1 S)$ which is then identified through its decay to $e^{+} e^{-}$or $\mu^{+} \mu^{-}$. These four photons need to be properly combined to determine the $\Upsilon(1 D)$ mass, especially in the phase of the potentially confusing cascade through the $\Upsilon(2 S)$, also shown in the level diagram. The histograms on the right demonstrate the $\Upsilon(1 D)$ mass, determined with each of two different methods for disentangling the photons.

used to extract the mass in the midst of photon backgrounds, and the figure also shows that both methods yield consistent values for the mass.

The results are consistent with the observation of a single state from the triplet, and that the state has $J=2$. We find

$$
M\left[\Upsilon\left(1^{3} D_{2}\right)\right]=10161.1 \pm 0.6 \pm 1.6
$$

This mass is one of the values used in comparing recent high precision unquenched Lattice QCD results to experiment [2].

\subsection{Radiative transitions in charmonium}

CLEO has made a number of measurements of both inclusive [22] and exclusive [23] radiative transitions of several transitions indicated in Fig. 6. In particular, by combining various inclusive and exclusive results, the following absolute branching ratios are determined for the $\chi_{c_{J}}$ :

$$
\begin{aligned}
& \mathscr{B}\left(\chi_{c_{0}} \rightarrow \gamma J / \psi\right)=2.0 \pm 0.2 \pm 0.2 \% \\
& \mathscr{B}\left(\chi_{c_{1}} \rightarrow \gamma J / \psi\right)=37.9 \pm 0.8 \pm 2.1 \% \\
& \mathscr{B}\left(\chi_{c_{2}} \rightarrow \gamma J / \psi\right)=19.9 \pm 0.5 \pm 1.2 \%
\end{aligned}
$$

These results are considerably higher than previous measurements for $J=0,1$. It came to this speaker's attention during the conference, that these rates are in fact calculable in Lattice QCD with significant precision. 


\section{Outlook}

CLEO-c continues to take data, and will do so for the next $\sim$ two years. Our results for $D$ meson decay are essentially limited by statistics, and an important goal is to improve that precision. All indications are that systematic error will not be a limiting factor for most of our measurements. Modifications to CESR will take place in Fall 2005 that are expected to boost the luminosity by a factor of two. The collaboration is currently discussing internally how best to exploit our remaining opportunities for data acquisition.

At the time of this writing, CLEO is taking data at a series of energies in the neighborhood of 4.0-4.2 GeV. A primary goal of these runs is to identify the optimum point for acquiring a large sample of $D_{s}$ mesons. Furthermore, even though it is not as clean as running at the $\psi(3770)$, there are still many relevant kinematic constraints that can be placed on events with extra pions or photons, in addition to those from the decay products of $D$ and $D_{s}$. Consequently, we are actively measuring the cross section for reactions such as $D^{\star} \bar{D}$ and $D^{\star} \bar{D}^{\star}$, as well as $D_{s}^{+} D_{s}^{-}$and $D_{s}^{\star} \bar{D}_{s}$.

The original CLEO-c proposal [8] emphasized the search for glueballs $\mathscr{G}$ in $J / \psi$ radiative decay. That is, the reaction $J / \psi \rightarrow \gamma \mathscr{G}$ is understood [24] to be a strong source for a glueball component in the isoscalar meson spectrum. There was also considerable experimental evidence at the time of the proposal, for a tensor glueball produced in this decay, at a mass near $2.2 \mathrm{GeV}$ [25]. There has been a significant shift in both the theoretical and experimental situations since that time, however, and the CLEO-c collaboration is in the midst of reevaluating our original plan to take data at the $J / \psi$. Other opportunities which may supplant this include taking a large sample at the $\psi(2 S)$, or investigating the recently observed $Y(4260)[26,27]$ which is a candidate for a gluonic $c \bar{c}$ hybrid meson [28].

In any case, CLEO is pleased to have had such a fruitful relationship with the Lattice QCD community. We look forward to continuing this extended collaboration.

\section{References}

[1] C. Davies, Lattice QCD - A guide for people who want results, hep-lat/0509046.

[2] HPQCD Collaboration, C. T. H. Davies et al., High-precision lattice QCD confronts experiment, Phys. Rev. Lett. 92 (2004) 022001, [hep-lat/ 0304004 ].

[3] I. Shipsey, Particle physics: Lattice window on strong force, Nature 427 (2004) 591-592.

[4] S. Dürr, Theoretical issues with staggered fermion simulations, hep-lat/ 0509026.

[5] K. Berkelman, A Personal History of CESR and CLEO. World Scientific, 2004.

[6] D. Peterson et al., The CLEO III drift chamber, Nucl. Instrum. Meth. A478 (2002) 142-146.

[7] M. Artuso et al., Construction, pattern recognition and performance of the CLEO III LiF-TEA RICH detector, Nucl. Instrum. Meth. A502 (2003) 91-100, [hep-ex/ 0209009 ].

[8] R. A. Briere et al., CLEO-c and CESR-c: A new frontier of weak and strong interactions, CLNS 01-1742, Cornell University, 2001.

[9] CLEO Collaboration, G. Bonvicini et al., Measuring $B\left(D^{+} \rightarrow \mu^{+} v\right)$ and the pseudoscalar decay constant $f_{D^{+}}$, Phys. Rev. D70 (2004) 112004, [hep-ex/ 0411050 ]. 
[10] CLEO Collaboration, M. Artuso, Improved measurement of $B\left(D^{+} \rightarrow \mu^{+} v\right)$ and the pseudoscalar decay constant $f_{D^{+}}$, hep-ex/0508057.

[11] D. Scora and N. Isgur, Semileptonic meson decays in the quark model: An update, Phys. Rev. D52 (1995) 2783-2812, [hep-ph/9503486].

[12] D. Melikhov and B. Stech, Weak form factors for heavy meson decays: An update, Phys. Rev. D62 (2000) 014006, [hep-ph/0001113].

[13] A. Khodjamirian, R. Ruckl, S. Weinzierl, C. W. Winhart, and O. I. Yakovlev, Predictions on $B \rightarrow \pi \bar{\ell} v_{\ell}, D \rightarrow \pi \bar{\ell} v_{\ell}$ and $D \rightarrow K \bar{\ell} v_{\ell}$ from QCD light-cone sum rules, Phys. Rev. D62 (2000) 114002 , [hep-ph/0001297].

[14] A. X. El-Khadra, A. S. Kronfeld, P. B. Mackenzie, S. M. Ryan, and J. N. Simone, The semileptonic decays $B \rightarrow \pi \ell v$ and $D \rightarrow \pi \ell v$ from lattice $Q C D$, Phys. Rev. $D 64$ (2001) 014502,

[hep-ph/0101023].

[15] Fermilab Lattice Collaboration, C. Aubin et al., Semileptonic decays of D mesons in three-flavor lattice QCD, Phys. Rev. Lett. 94 (2005) 011601, [hep-ph/ 040830 6].

[16] CLEO Collaboration, G. S. Huang et al., Study of semileptonic charm decays $D^{0} \rightarrow \pi^{-} \ell^{+} v$ and $D^{0} \rightarrow K^{-} \ell^{+} v$, Phys. Rev. Lett. 94 (2005) 011802, [hep-ex/ 0407035 ].

[17] CLEO Collaboration, T. E. Coan et al., Absolute branching fraction measurements of exclusive $D^{0}$ semileptonic decays, hep-ex/0506052.

[18] CLEO Collaboration, G. S. Huang et al., Absolute branching fraction measurements of exclusive $D^{+}$ semileptonic decays, hep-ex/0506053.

[19] CLEO Collaboration, J. L. Rosner et al., Observation of $h_{c}\left({ }^{1} P_{1}\right)$ state of charmonium, Phys. Rev. Lett. 95 (2005) [hep-ex/ 0505073$].$

[20] CLEO Collaboration, P. Rubin et al., Observation of the ${ }^{1} P_{1}$ state of charmonium, hep-ex/0508037.

[21] CLEO Collaboration, G. Bonvicini et al., First observation of a $\Upsilon(1 D)$ state, Phys. Rev. D70 (2004) 032001, [hep-ex/0404021].

[22] CLEO Collaboration, S. B. Athar et al., Photon transitions in $\psi(2 S)$ decays to $\chi_{c J}(1 P)$ and $\eta_{c}(1 S)$, Phys. Rev. D70 (2004) 112002, [hep-ex/ 0408133 ].

[23] CLEO Collaboration, N. E. Adam, Branching fractions for $\psi(2 S) \rightarrow J / \psi$ transitions, Phys. Rev. Lett. 94 (2005) 232002, [hep-ex/0503028].

[24] F. E. Close, G. R. Farrar, and Z.-p. Li, Determining the gluonic content of isoscalar mesons, Phys. Rev. D55 (1997) 5749-5766, [hep-ph/9610280].

[25] S. Eidelman et al., Review of Particle Physics, Physics Letters B 592 (2004) 1+.

[26] BABAR Collaboration, B. Aubert et al., Observation of a broad structure in the $\pi^{+} \pi^{-} J / \psi$ mass spectrum around 4.26-GeV/c2 hep-ex/0506081.

[27] BABAR Collaboration, B. Aubert et al., Study of $J / \psi \pi^{+} \pi^{-}$states produced in $B^{0} \rightarrow J / \psi \pi^{+} \pi^{-} K^{0}$ and $B^{-} \rightarrow J / \psi \pi^{+} \pi^{-} K^{-}$, hep-ex/0507090.

[28] F. E. Close and P. R. Page, Gluonic charmonium resonances at BaBar and Belle?, hep-ph/0507199. 